Familienanamnese und der deutlichen Größenzunahme war jedoch ein fettig degeneriertes hepatocelluläres Karzinom (HCC) nicht auszuschließen. In der MRT-Untersuchung zeigte der Tumor eine kapselartige, relativ glatte Begrenzung. Die Untersuchung im $\mathrm{T}_{1}$-gewichteten Modus mit und ohne Fettsuppression (Abb. $\mathbf{2} \mathbf{a}, \mathbf{b}$ ) ermöglichte den eindeutigen Nachweis größerer Fettanteile. In der $\mathrm{T}_{2}$-gewichteten, fettgesättigten Sequenz zeigte sich ein inhomogener, deutlich signalhyperintenser Tumor mit triangulärem, lipomatösem Anteil (ohne Abb.).

Darüber hinaus fand sich ein $1,5 \mathrm{~cm}$ großer Tumor am Unterpol der rechten Niere (Abb.3). Dieser reicherte teilweise KM an und zeigte zusätzlich deutliche hypodense Anteile. In einer ergänzenden CT-Dünnschichtuntersuchung der Nieren ohne Kontrastmittel enthielt der Befund negative Dichtewerte bis - 49 HE. Die sonographische Untersuchung ergab mit einer homogen echogenen Binnenstruktur und scharfer Herdbegrenzung ebenfalls den typischen Befund eines AML. Aufgrund der zunehmenden klinischen Symptomatik, der Größenprogredienz und der unklaren Dignität des Prozesses wurde eine Hemihepatektomie links durchgeführt.

\title{
Koinzidenz eines Angiomyolipoms der Leber und der Niere
}

Angiomyolipome (AML) der Leber sind seltene, gutartige Tumoren, die einen variablen Anteil an Fettgewebe, Blutgefäßen und glatter Muskulatur enthalten. Sie können bei tuberöser Hirnsklerose im Zusammenhang mit AML der Nieren auftreten.

Wir beschreiben den Fall des parallelen Auftretens eines großen AML der Leber und eines zusätzlichen kleinen AML der rechten Niere. Klinisch lagen keine Anhaltspunkte für eine tuberöse Hirnsklerose vor.

\section{Fallbericht}

Bei einem 42-jährigen Patienten war 1996 nach einem stumpfen Bauchtrauma sonographisch und computertomographisch ein ca. $6 \times 5 \times 4 \mathrm{~cm}$ großer, artdiagnostisch unklarer Lebertumor festgestellt worden. Der Patient verweigerte damals eine weitere Abklärung. Es bestanden keine Zeichen einer Leberzir- rhose, der alpha1-Fetoprotein-Spiegel im Serum war im Normbereich. Im September 1998 klagte der Patent bei Klinikaufnahme über Oberbauchschmerzen, retrosternales Druckgefühl und einen Leistungsknick in den letzten Monaten. Eine auswärtig durchgeführte CT-Untersuchung zeigte eine deutliche Größenzunahme des Leberbefundes seit 1996 auf nun $15 \times 10 \times 10 \mathrm{~cm}$. Der Patient wurde zur operativen Entfernung des Tumors in unsere Klinik überwiesen.

Die erneut durchgeführte Spiral-CT-Untersuchung des Abdomens (nativ, in arterieller und portovenöser Phase) zeigte den bekannten Lebertumor (Abb.1), der die Segmente II-IV komplett einnahm und am Rand teils flächig, teils nodulär intensiv Kontrastmittel (KM) aufnahm. Die Binnenstruktur war inhomogen mit großflächigen, exzentrisch gelegenen lipomatösen Anteilen. Es wurde die Verdachtsdiagnose eines AML gestellt. Aufgrund einer positiven
Die Histologie ergab ein AML ohne Anhalt für Malignität. Nachträglich durchgeführte CT- und MR-Untersuchungen des Schädels zeigten keinen Hinweis auf eine tuberöse Sklerose.

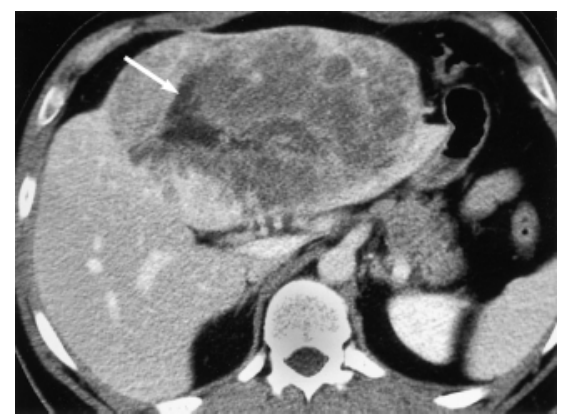

Abb. 1 Computertomogramm mit Kontrastmittel i.v. Großer Tumor im linken Leberlappen mit deutlich hypodensen Anteilen (bis -50 Hounsfield-Einheiten; Pfeil).

\section{Diskussion}

Angiomyolipome, Angiomyelolipome und Angiomyomyelolipome der Leber bilden eine Klasse von gutartigen mesenchymalen Tumoren, die aus unter- 
a

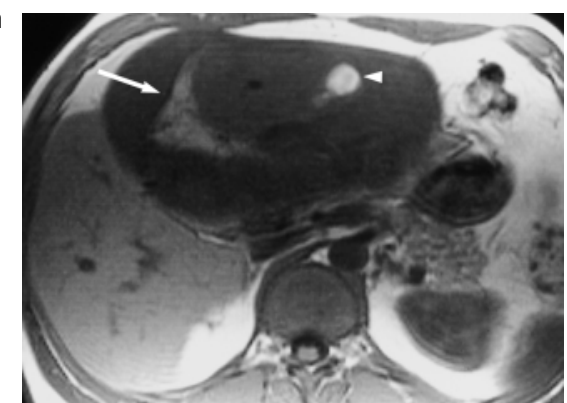

b

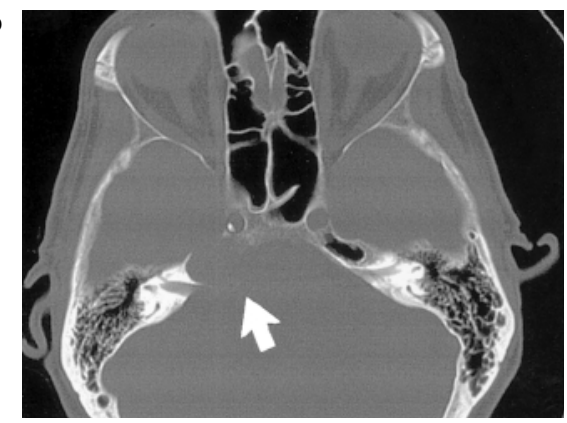

Abb. 2 (a) Die axiale, $T_{1}$-gewichtete native Kernspintomographie ohne Fettsuppression zeigt einen hyperintensen triangulären Anteil des Tumors (Pfeil) sowie zusätzlich eine rundliche, stark hyperintense Läsion (Pfeilspitze). (b) In der $\mathrm{T}_{1}$-gewichteten nativen Untersuchung mit Fettsuppression verlieren die Fettanteile des Tumors deutlich an Signal (Pfeil). Der hämorrhagische Herd (Pfeilspitze) bleibt unverändert, das Signalverhalten spricht für die Präsenz von Methämoglobin.

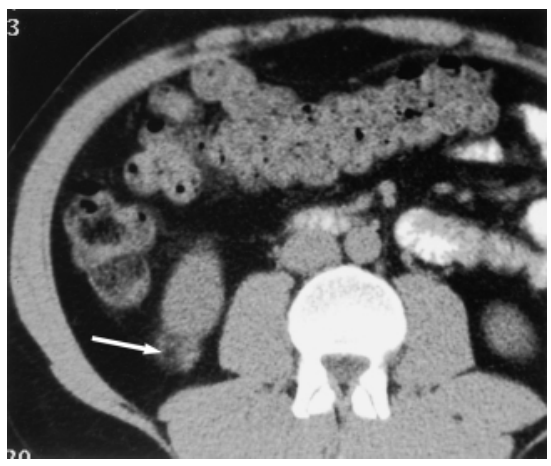

Abb. 3 Computertomogramm nativ. Tumor am Unterpol der rechten Niere mit negativen Dichtewerten bis - 49 Hounsfield-Einheiten; (Pfeil).

schiedlich gewichteten Anteilen von Blutgefäßen, Fettzellen, glatter Muskulatur und teilweise auch knochenmarkähnlichem, blutbildendem Gewebe bestehen. Über die Entstehung von Angiomyolipomen und ihr Wachstumsverhalten gibt es nur wenige Erkenntnisse, wobei einige Autoren (Irie $\mathrm{H}$ et al., J Com Ass Tom 1999;23(2):310) eine Größen- a progredienz, wie sie auch in unserem Fall zu beobachten war, beschreiben.

Das klinische Bild hepatischer AML ist unspezifisch und meist abhängig von der Größe des Tumors. In der uns bekannten Literatur finden sich Tumorgrößen von 0,2 bis $28 \mathrm{~cm}$ (Strotzer M et al., Eur Radiol 1999; 9:259). Sola et al. (Clin Imaging 1996; 20:99) schätzen die Gefahr einer spontanen Blutung, wie sie von renalen b AML bekannt ist, auch bei größeren Befunden trotz des Reichtums an vaskulären Strukturen mit degenerierten Gefäßwänden und kleinen Aneurysmen als sehr gering ein. Konkrete Therapieempfehlungen wie etwa bei renalen AML gibt es nicht. Die maligne Transformation eines AML der Leber wurde bisher nicht beschrieben. Ebenso fehlen bisher Veröffentlichungen, ob hepatische AMLs zur „Metastasierung“ neigen, analog der bei renalen AMLs auftretenden Involvierung regionaler Lymphknoten (was von einigen Autoren eher als multifokales Auftreten des AML denn als Metastasierung gesehen wird (Sola et al.). Irie et al. empfehlen eine Verlaufskontrolle mittels bildgebender Verfahren, da mit der Tumorgröße auch die Gefäßanomalien und somit die Blutungsgefahr zunehmen. Bisher ist nur in einem Fall die Ruptur eines knapp $8 \mathrm{~cm}$ messenden, hepatischen AML publiziert.

Bei der Nachuntersuchung von 50 Patienten mit renalen AML fanden Roberts JL et al. (Radiology $1986 ; 158: 613$ ) fünf Fälle mit simultan nachweisbarem AML der Leber. Genau wie AML der Niere können auch solche der Leber mit tuberöser Hirnsklerose vergesellschaftet sein. In einer Literatur-Recherche von 39 beschriebenen Fällen von AML der Leber waren 12 mit tuberöser Hirnsklerose assoziiert (Strotzer et al.). In dem von uns vorgestellten Fall gab es dafür keine Hinweise.

Im Ultraschall stellt sich ein AML typischerweise als echoreiche Formation dar (Roberts et al.). Die hohe Echogenität beruht dabei vermutlich auf der hohen Anzahl von Blutgefäßen und der Anwesenheit von Fettgewebe. Da der Gehalt an Fett zwischen 10 und weit über 50\% liegen kann, ist der Nachweis von Fettanteilen mittels CT oder MR nicht immer möglich.

Nach i.v. Applikation von Kontrastmittel zeigen AML eine unterschiedlich ausgeprägte Kontrastmittelaufnahme im CT und in der MRT. Aufgrund ihres Kontrastmittelverhaltens können hepatische AML eine fokal noduläre Hyperplasie (FNH) vortäuschen (Strotzer et al.).

Darüber hinaus müssen bei der Differenzialdiagnose von fetthaltigen Leberläsionen sowohl fokale fettige Infiltrationen der Leber als auch Lipome, Adenome, Myelolipome, Liposarkome (LSA) und Metastasen von Liposarkomen oder Teratokarzinomen (TCA) in Betracht gezogen werden. Bisher ist nur ein einziger Fall eines primären LSA in der Leber beschrieben und Lebermetastasen eines LSA sind durch das Vorliegen eines Primärtumors und ihr meist multizentrisches Auftreten von einem AML zu differenzieren. TCA und ihre Metastasen lassen sich durch das Vorliegen von Verkalkungen unterscheiden.

Ebenfalls muss an eine fettige Umwandlung innerhalb eines HCC gedacht werden, obwohl diese bei nicht asiatischen Patienten sehr selten auftritt.

Nach Tsui et al. (AJ Surg Path 1999; 23(1): 34) hat sich der primär in der Melanomdiagnostik verwendete monoklonale Antikörper HMB-45 als ein hervorragender Marker zur histopathologischen Identifizierung eines AML erwiesen. Von den bisher mehr als 30 überprüften AML waren alle HMB- 45 positiv und konnten dadurch von anderen Tumoren wie LSA TCA oder HCC differenziert werden

Im Gegensatz zum Vorgehen beim renalen AML empfehlen wir daher bei V. a. ein hepatisches AML, aufgrund der zahlreichen DD von fetthaltigen Tumoren der Leber, die Biopsie und anschließende histologische sowie immunhistochemische Abklärung des Befundes. Hierbei ist auf die mögliche Blutungsgefahr vor allem bei oberflächlich liegenden Herden zu achten.

T. Finkenzeller, M. Völk, M. Strotzer, Regensburg 